\title{
Antimicrobial cathelicidin peptide LL-37 induces NET formation and suppresses the inflammatory response in a mouse septic model
}

\author{
HIROSHI HOSODA ${ }^{1}$, KAHO NAKAMURA ${ }^{1}$, ZHONGSHUANG HU ${ }^{1}$, HIROSHI TAMURA ${ }^{1,2}$, JOHANNES REICH ${ }^{3}$, \\ KYOKO KUWAHARA-ARAI ${ }^{4}$, TOSHIAKI IBA ${ }^{5}$, YOKO TABE $^{6}$ and ISAO NAGAOAKA ${ }^{1}$ \\ ${ }^{1}$ Department of Host Defense and Biochemical Research, Juntendo University, Graduate School of Medicine, Tokyo 113-8421; \\ ${ }^{2}$ Laboratory Program Support (LPS) Consulting Office, Tokyo 160-0023, Japan; ${ }^{3}$ Microcoat Biotechnologie GmbH, \\ D-82347 Bernried, Germany; Departments of ${ }^{4}$ Microbiology, ${ }^{5}$ Emergency and Disaster Medicine, \\ ${ }^{6}$ Clinical Laboratory Medicine, Juntendo University, Graduate School of Medicine, Tokyo 113-8421, Japan
}

Received March 28, 2017; Accepted July 25, 2017

DOI: $10.3892 / \mathrm{mmr} .2017 .7267$

\begin{abstract}
LL-37 is the only known member of the cathelicidin family of antimicrobial peptides in humans. In addition to its broad spectrum of antimicrobial activities, LL-37 may modulate various inflammatory reactions. The authors previously revealed that LL-37 improves the survival of a murine cecal ligation and puncture (CLP) sepsis model. In the present study, the mechanism for the protective action of LL-37 was elucidated using the CLP model, focusing on the effect of LL-37 on the release of neutrophil extracellular traps (NETs). The results indicated that the intravenous administration of LL-37 suppressed the increase of damage-associated molecular patterns (DAMPs), including histone-DNA complex and high-mobility group protein 1 , in addition to interleukin-1 $\beta$, tumor necrosis- $\alpha$ and soluble triggering receptor expressed on myeloid cells (TREM)-1 in plasma and peritoneal fluids. Notably, LL-37 significantly suppressed the decrease of mononuclear cell number in blood, and the increase of polymorphonuclear cell (neutrophil) number in the peritoneal cavity during sepsis. Furthermore, LL-37 reduced the bacterial burden in blood and peritoneal fluids. Notably, LL-37 increased the level of NETs (myeloperoxidase-DNA complex) in plasma and peritoneal fluids. In addition, it was verified that LL-37 induces the release of NETs from neutrophils, and NETs possess the bactericidal activity. Overall, these observations suggest that LL-37 improves the survival of CLP septic mice by possibly suppressing the inflammatory responses as evidenced by the inhibition of the increase of cytokines, soluble TREM-1 and DAMPs (host cell death) and the
\end{abstract}

Correspondence to: Professor Isao Nagaoka, Department of Host Defense and Biochemical Research, Juntendo University, Graduate School of Medicine, 2-1-1 Hongo, Bunkyo-Ku, Tokyo 113-8421, Japan

E-mail: nagaokai@juntendo.ac.jp

Key words: LL-37, cathelicidin, antimicrobial peptides, sepsis, neutrophil extracellular traps, cytokines, TREM-1, DAMPs alteration of inflammatory cell numbers, and bacterial growth via the release of NETs with bactericidal activity.

\section{Introduction}

Sepsis is a systemic inflammatory response syndrome (SIRS) caused by infection (1), and still a most common cause of death in non-coronary intensive care unit (2). As the major mechanism for the susceptibility to sepsis, dysregulated inflammatory reactions are considered; however, anti-inflammatory cytokine therapies are inefficient in clinical trials (2-4). Recently, much attention has been focused on the mechanism of host cell death, which develops during sepsis and contributes to the dysregulated inflammatory reaction, immunosuppression and organ failure in sepsis $(5,6)$.

Antimicrobial peptides represent the first line of defense against invading pathogens (7). Cathelicidin family of antimicrobial peptides has been identified in various mammalian species, and LL-37 is the only known human cathelicidin, primarily produced by epithelial cells and neutrophils (7). In addition to its antimicrobial activity, LL-37 exhibits diverse biological activities, including regulation of inflammatory responses $(7,8)$. Importantly, we previously revealed that LL-37 inhibits the spontaneous apoptosis of neutrophils via purinergic receptor $\mathrm{P} 2 \mathrm{X}_{7}$ and formyl-peptide receptor-like 1 (FPRL1), and reduces the lipopolysaccharide (LPS)-induced apoptosis of endothelial cells by neutralizing the action of LPS $(9,10)$, suggesting that LL-37 is involved in the modulation of cell death. Furthermore, LL-37 exerted the protective actions on murine endotoxin shock by blocking the binding of LPS to CD14-positive cells and suppressing the production of cytokines by these cells (11).

Neutrophils, the most abundant leukocytes in humans, protect the host against invading microorganisms as an essential part of the innate immune system $(12,13)$. Neutrophils exhibit antimicrobial potential by phagocytosing microorganisms and releasing antimicrobial agents (e.g., reactive oxygen species and antimicrobial peptides) (12). Moreover, upon exposure to bacteria or sterile stimuli, neutrophils undergo NETosis (a type of programmed cell death) and release neutrophil extracellular traps (NETs) (14). Notably, NETs are functioning as an 
antimicrobial mechanism, based on the fact that NETs can trap microorganisms and exert bactericidal activity by the action of NET-associated components [e.g., antimicrobial peptides and histone] (15-17). In addition, the major components of NETs (histone, DNA and granule proteins) are recognized as damage-associated molecular patterns (DAMPs), which trigger inflammatory signals to induce inflammation, cell death and organ failure (18-22); for example, extracellular histones mediate mortality in mouse fatal liver injury (21).

Recently, we have demonstrated that the intravenous administration of LL-37 improved the survival of a murine cecal ligation and puncture (CLP) sepsis model (23). Notably, it is demonstrated that LL-37 induces NETs release from the neutrophils in vitro (24). Thus, we hypothesized that LL-37 may induce the release of NETs from neutrophils in vivo and protect mice from CLP-induced sepsis. In this study, to test this hypothesis, we intravenously administered LL-37 into a CLP septic model of mice, and evaluated the effect of LL-37 on the levels of inflammatory cytokines (TNF- $\alpha$ and IL-1 $\beta$ ), triggering receptor expressed on myeloid cells (TREM)-1, DAMPs [histone-DNA complex and high-mobility group protein 1 (HMGB1)] and bacterial burdens as well as NETs (determined as myeloperoxidase-DNA complex) in plasma (blood) and peritoneal fluids of CLP septic mice. The results indicated that LL-37 protects CLP septic mice by possibly suppressing the inflammatory responses (the increase of TNF- $\alpha$, IL-1 $\beta$, TREM-1 and DAMPs) and bacterial growth via the induction of NETs.

\section{Materials and methods}

Reagents. A 37-mer peptide of hCAP18 (LL-37; L'LGDFFRKSKEKIGKEFKRIVQRIKDFLRNLVPRTES ${ }^{37}$ ) was synthesized by the solid-phase method on a peptide synthesizer (model PSSM-8; Shimadzu, Kyoto, Japan) by F-moc chemistry and purified as described previously $(19,25)$. Phorbol 12-myristate 13-acetate (PMA) was purchased from Calbiochem (product no. 524400; La Jolla, CA, USA). Anti-myeloperoxidase (MPO) mAb (product no. 392105) was purchased from R\&D Systems, Inc. (Minneapolis, MN, USA).

Mice. Male BALB/c mice (23 g, 8 weeks old; Sankyo Labo Service, Inc., Tokyo, Japan) were used in the experiments. Mice were bred under specific-pathogen-free conditions, and housed in temperature-controlled, air-conditioned facilities with 12/12-h light/dark cycles with ad libitum access to food and water. All experiments were approved by the Ethics Committee for the Use of Laboratory Animals of Juntendo University, Graduate School of Medicine (permission no. 280014).

CLP septic mouse model. The CLP procedure was performed according to the general guidelines (26), as described previously (23). Mice were anesthetized using 2\% isoflurane (product no. 099-06571; Wako Pure Chemical Industries, Osaka, Japan) in oxygen with a delivery rate of $0.21 / \mathrm{min}$. After disinfection of the abdomen with $70 \%$ ethanol, a 1-cm midline laparotomy was performed and the cecum was exposed. Cecal contents were massaged to the tip, and the distal $0.5 \mathrm{~cm}$ of the tip was ligated with 3-0 silk sutures (product no. GA03SW;
Alfresa Pharma Corporation, Osaka, Japan). A 21-G needle was used to perforate the ligated portion of the cecum once in a through-and-through manner, and a small amount of stool (1 mm in length) was extruded. The cecum was returned to the abdominal cavity, and the abdomen and skin were respectively closed using 3-0 nylon suture (product no. ER1203NA45-KF2; Alfresa Pharma Corporation). Following the surgery, $1 \mathrm{ml}$ of saline was subcutaneously administered in the neck for rehydration.

In the Sham group, mice underwent the same procedure but without the cecal ligation and the puncture. In the Sham group or LL-37 group, saline (100 $\mu \mathrm{l})$ or LL-37 (2 $\mu \mathrm{g}$ in $100 \mu \mathrm{l}$ saline) was administered intravenously per mouse, respectively, just before CLP, and then the CLP procedure was immediately performed. Thereafter, the survival rates of the mice were monitored.

Preparation of peritoneal fluids and blood samples. At the indicated period $(20 \mathrm{~h}$ ) after the surgery, $4 \mathrm{ml}$ of cold phosphate-buffered saline (PBS) were intraperitoneally injected, and the peritoneal fluids $(\sim 4 \mathrm{ml})$ were collected after gentle massage for $1 \mathrm{~min}$. In addition, blood was obtained by cardiac puncture in heparinized syringe, and plasma was prepared by centrifugation of blood at $700 \mathrm{x}$ g for $20 \mathrm{~min}$ at room temperature.

Quantification of cytokine, HMGB1 and TREM-1 levels. Plasma and peritoneal fluids were used for the assays of cytokines, HMGB1 and TREM-1. Cytokines (TNF- $\alpha$ and IL-1 $\beta$ ) were determined using commercially available mouse TNF alpha ELISA Ready-SET-Go! and Mouse IL-1 beta ELISA Ready-SET-Go! (product nos. 88-7324-77 and 88-7013-77; eBioscience, San Diego, CA, USA), according to the manufacturer's instructions. TREM-1 and HMGB1 were determined using a commercially available mouse TREM-1 Duo set ELISA (product no. DY1187; R\&D Systems, Inc.) or HMGB1 ELISA kit (product no. 326054329; Shino-Test Corporation, Kanagawa, Japan), according to the manufacturers' instructions, respectively. Concentrations of cytokines, TREM-1 and HMGB1 were expressed as $\mathrm{pg} / \mathrm{ml}, \mathrm{pg} / \mathrm{ml}$ and $\mathrm{ng} / \mathrm{ml}$ of plasma and peritoneal fluids collected, respectively.

Quantification of histone-DNA complex level. Histone-DNA complex was determined in plasma and peritoneal fluids using a commercially available Cell Death Detection ELISA ${ }^{\text {PLUS }}$ (product no. 11774425001; Roche Applied Science, Mannheim, Germany), according to the manufacturer's instructions. Concentrations of histone-DNA complex were expressed as $\mathrm{mU} / \mathrm{ml}$ of plasma and peritoneal fluids collected, using a positive control histone-DNA complex contained in the ELISA kit (mU $=$ absorbance of $10^{-3}$ at $405 \mathrm{~nm}$ ).

Quantification of mononuclear cells and polymorphonuclear cells in blood and peritoneal fluids. Blood and peritoneal fluids were collected, as described above, and diluted 10-fold with Turk's stain solution (product no. 37212-74; Nacalai Tesque, Inc., Kyoto, Japan). The numbers of mononuclear cells and polymorphonuclear cells were counted based on the shape of nuclei, using a hemocytometer under the microscope. The number of mononuclear cells and polymorphonuclear 
cells were expressed as cells/ml of blood and peritoneal fluids collected.

Quantification of bacterial burden. Blood and peritoneal fluid samples were serially diluted in PBS. Diluted samples (100 $\mu \mathrm{l})$ were plated on Trypto-Soya agar plates (product no. 05516; Nissui Pharmaceutical, Tokyo, Japan), and the plates were incubated for $20 \mathrm{~h}$ at $37^{\circ} \mathrm{C}$. The colony forming units (CFU) were counted and corrected for the dilution factor. The number of bacteria was expressed as $\mathrm{CFU} / \mathrm{ml}$ of blood and peritoneal fluids collected.

Quantification of MPO-DNA complex level. MPO-DNA complex was determined in plasma and peritoneal using a commercially available Cell Death Detection ELISA ${ }^{\text {PLUS }}$ (product no. 11774425001; Roche Applied Science) and anti-MPO mAb (no. 392105; R\&D Systems, Inc.) as a capture antibody. Briefly, a 96-well half area plate (product no. 3690; Corning Life Sciences, Lowell, MA, USA) was coated with by 250 ng/well anti-MPO mAb (no. 392105; R\&D Systems, Inc.) overnight at $4^{\circ} \mathrm{C}$. After washing 3 times, $10 \mu \mathrm{l}$ of approximately diluted samples were added to the wells with $40 \mu \mathrm{l}$ of incubation buffer containing a peroxidase-labeled anti-DNA monoclonal antibody (MAC-33; Cell Death Detection ELISA ${ }^{\text {PLUS }}$ ). The plate was incubated for $2 \mathrm{~h}$ with shaking at $300 \mathrm{rpm}$ at room temperature. After washing 3 times, $50 \mu$ l peroxidase substrate (ABTS; Cell Death Detection ELISA ${ }^{\text {PLUS }}$ ) was added, and absorbance at $405 \mathrm{~nm}$ was measured after 30-min incubation at room temperature in the dark.

To prepare a standard for NETs containing MPO-DNA complex, mouse bone marrow-derived neutrophils were prepared and stimulated, as described previously (24,27-29). In brief, bone marrow neutrophils were isolated from femurs and purified by a Percoll density gradient (product no. 17089101, GE Healthcare, Uppsala, Sweden). Cells $\left(10^{6}\right)$ were plated in the 24-well plates in $400 \mu \mathrm{l}$ RPMI-1640 (product no. 30264-85; Nacalai Tesque, Inc.,) supplemented with $1 \%$ fetal bovine serum (FBS, product no. 172012; Nichirei Biosciences Inc., Tokyo, Japan) and stimulated with $5 \mu \mathrm{M}$ LL-37 for $4 \mathrm{~h}$. Then, the cells were treated with $2 \mathrm{U} / \mathrm{ml}$ DNase I (product no. 2270A; Takara Bio, Kyoto, Japan) for $15 \mathrm{~min}$ at room temperature to allow the limited DNA digestion of NETs, and then DNase digestion was stopped by adding $5 \mathrm{mM}$ EGTA $(28,29)$. Thereafter, the reaction mixtures were centrifuged at $500 \mathrm{x}$ g for 5 min at $4^{\circ} \mathrm{C}$, and the supernatants were collected as a standard for NETs $\left(10^{6}\right.$ cell equivalents $\left./ 400 \mu \mathrm{l}\right)$. MPO-DNA levels were corrected using a standard for NETs ( 1 unit containing $2.5 \times 10^{4}$ cell equivalents $/ 10 \mu \mathrm{l}$ ), as described above. Concentrations of MPO-DNA complex were expressed as U/ml of plasma and peritoneal fluids collected.

Induction of NET release and bactericidal activity of NETs. To induce the release of NETs from neutrophils, NETs were prepared by stimulating mouse bone marrow-derived neutrophils $\left(10^{6}\right)$ with $100 \mathrm{nM}$ PMA or $5 \mu \mathrm{M} \mathrm{LL}-37$ for $4 \mathrm{~h}$ at $37^{\circ} \mathrm{C}$ in $400 \mu \mathrm{l}$ RPMI-1640 supplemented with $1 \% \mathrm{FBS}$. Alternatively, neutrophils were incubated without PMA or LL-37 for $4 \mathrm{~h}$ at $37^{\circ} \mathrm{C}$ (unstimulated neutrophils). Then, the supernatants were recovered by centrifugation at $500 \mathrm{x} \mathrm{g}$ for $5 \mathrm{~min}$ at $4^{\circ} \mathrm{C}$, and assayed for the level of MPO-DNA complex, as described above. Furthermore, bactericidal activity of NETs was assayed; $10 \mu 1$ supernatants from unstimulated or PMA-stimulated neutrophils were added to $10 \mu \mathrm{l}$ of $E$. coli (TOP10, product no. C404003; Invitrogen; Thermo Fisher Scientific, Inc., Waltham, MA, USA) $\left(10^{7}\right.$ cells $)$ in LB broth (product no. 12780-052; Gibco-BRL; Thermo Fisher Scientific, Inc.) and incubated at room temperature for $30 \mathrm{~min}$. Mixtures were appropriately diluted, and samples $(100 \mu \mathrm{l})$ were plated on Trypto-Soya agar plates, and the plates were incubated for $20 \mathrm{~h}$ at $37^{\circ} \mathrm{C}$. The colony forming units (CFU) were counted and corrected for the dilution factor.

Statistical analysis. Data are shown as mean \pm standard deviation (SD). Statistical analyses were performed using the one-way analysis of variance (ANOVA), followed by Bonferroni's multiple comparison test (GraphPad Prism, version 5.0b; GraphPad Software, Inc., San Diego, CA, USA). A p-value $<0.05$ was considered to indicate a statistically significant differences.

\section{Results}

Effect of LL-37 administration on the levels of cytokines and TREM-1 in septic mice. Previously, we revealed that intravenous administration of LL-37 (2 $\mu \mathrm{g} / \mathrm{mouse})$ significantly improves the survival of CLP septic mice (23). In the present study, we confirmed that the survival rate in the CLP group $(47 \pm 50 \%, \mathrm{n}=17)$ was significantly lower than that in the Sham group $(100 \%, \mathrm{n}=7, \mathrm{P}<0.05)$ at 1 day after the surgery, and importantly the administration of LL-37 significantly improved the survival rate to $81 \pm 39 \%(n=16)(P<0.05)$. Thus, to elucidate the mechanism for the protective action of LL-37 on the septic mice, we first measured the levels of inflammatory cytokines and soluble TREM-1, a biomarker for sepsis (30-32) in CLP mice, and evaluated the effect of LL-37 administration on levels of these molecules in plasma and peritoneal fluids. TNF- $\alpha$ was detected in plasma and peritoneal fluids in the Sham group, and significantly increased in the CLP group $(\mathrm{P}<0.001)$ (Fig. 1A and B). Interestingly, LL-37 inhibited the increase of TNF- $\alpha$ levels both in plasma and peritoneal fluids $(\mathrm{P}<0.05)$.

Similarly, IL-1 $\beta$ was detected in plasma and peritoneal fluids in the Sham group, and significantly increased in the CLP group $(\mathrm{P}<0.001)$ (Fig. 1C and D); LL-37 inhibited the increase of IL-1 $\beta$ levels both in plasma and peritoneal fluids $(\mathrm{P}<0.05)$.

Furthermore, soluble TREM-1 was hardly detected in plasma and peritoneal fluids in the Sham group, but significantly increased in plasma and peritoneal fluids in the CLP group $(\mathrm{P}<0.001)$ (Fig. 1E and F). Importantly, LL-37 suppressed the increase of TREM-1 levels both in plasma and peritoneal fluids $(\mathrm{P}<0.05)$.

Effect of LL-37 administration on the levels of DAMPs in septic mice. During the course of sepsis-associated cell death, DAMPs (such as DNA, histones and HMGB1) are released from host cells and act as proinflammatory mediators (33-35). Thus, we next measured the levels of HMGB1 and histone-DNA complex in CLP mice, and evaluated the effect of LL-37 administration on these levels. HMGB1 was detected 
A
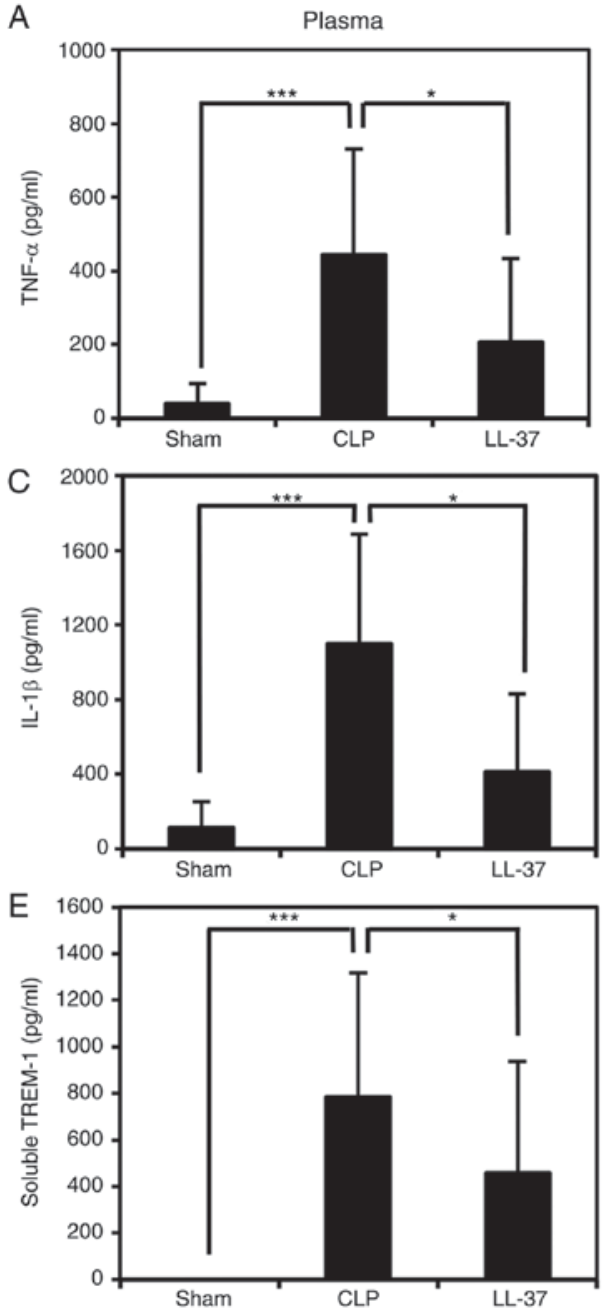

B
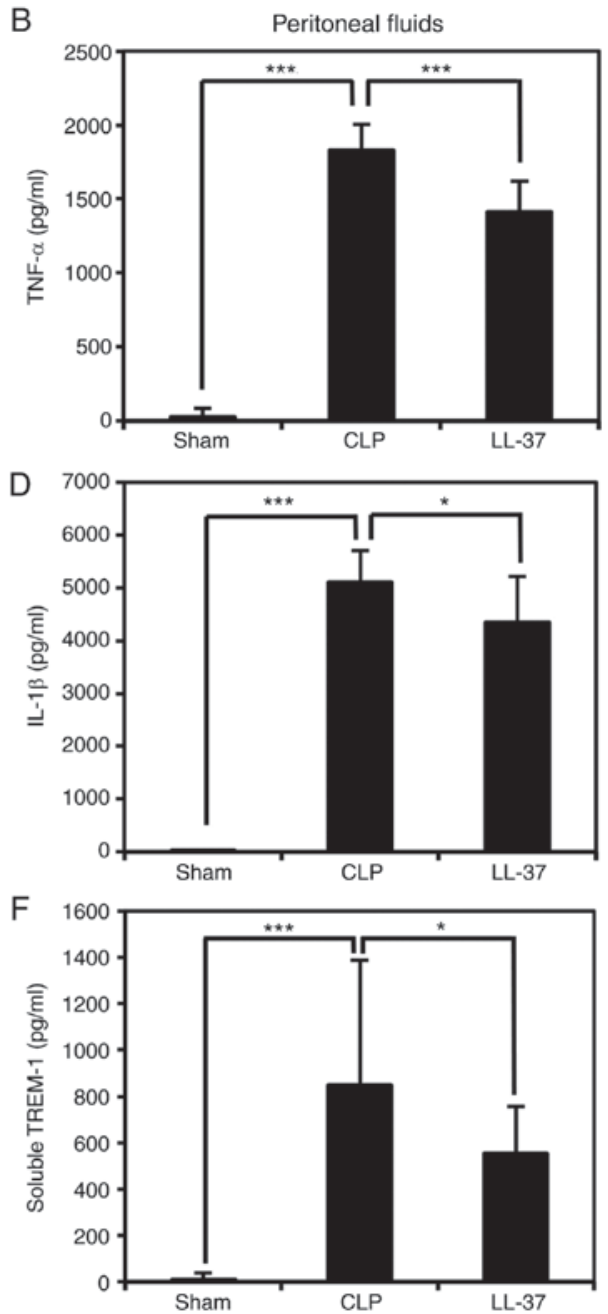

Figure 1. Effect of LL-37 administration on the levels of TNF- $\alpha$, IL-1 $\beta$ and soluble TREM-1 in plasma and peritoneal fluids. Plasma and peritoneal fluids were collected from mice of Sham, CLP and LL-37 groups at $20 \mathrm{~h}$ after the surgery, and assayed for TNF- $\alpha$, IL-1 $\beta$ and soluble TREM-1. (A and B) TNF- $\alpha$ levels in plasma and peritoneal fluids, respectively; (C and D) IL-1 $\beta$ levels in plasma and peritoneal fluids, respectively; (E and F) TREM-1 levels in plasma and peritoneal fluids, respectively. Concentrations of cytokines and TREM-1 are expressed as pg/ml of plasma and peritoneal fluids collected ( 4 ml). Data are the mean $\pm \mathrm{SD}$ of $7-19$ separate experiments, and values are compared among Sham, CLP and LL-37 groups. $\mathrm{P}<0.05$, ${ }^{* * *} \mathrm{P}<0.001$. TREM, triggering receptor expressed on myeloid; CLP, cecal ligation and puncture; IL, interleukin; TNF, tumor necrosis factor.

in plasma and peritoneal fluids in the Sham group, and significantly increased in the CLP group $(\mathrm{P}<0.001)$ (Fig. 2A and B). LL-37 inhibited the increase of HMGB1 levels both in plasma and peritoneal fluids $(\mathrm{P}<0.05)$.

Similarly, histone-DNA complex was detected in plasma and peritoneal fluids in the Sham group, and significantly increased in the CLP group ( $\mathrm{P}<0.001)$ (Fig. 2C and D); LL-37 inhibited the increase of histone-DNA complex both in peritoneal fluids $(\mathrm{P}<0.05)$ and plasma, although the inhibition by LL-37 was not significant in plasma.

Effect of LL-37 administration on number of white blood cells and peritoneal cells in septic mice. During infection, the numbers of white blood cells are changed, and inflammatory cells are recruited to the site of infection $(36,37)$. Thus, we evaluated the effect of LL-37 administration on the number of white blood cells and recruited cells in the peritoneal cavity of CLP mice. In blood, the number of mononuclear cells was markedly decreased in the CLP group $(\mathrm{P}<0.001)$, but was significantly increased in the LL-37 group compared with the CLP group $(\mathrm{P}<0.05)$ (Fig. 3A). In contrast, the number of polymorphonuclear cells was not essentially changed among Sham, CLP and LL-37 groups.

In the peritoneal cavity, the numbers of polymorphonuclear cells (mostly neutrophils) was markedly increased in the CLP group $(\mathrm{P}<0.001)$, but was significantly decreased in the LL-37 group compared with the CLP group $(\mathrm{P}<0.01)$ (Fig. 3B). In contrast, the number of mononuclear cells (mostly macrophages) was not significantly changed among Sham, CLP and LL-37 groups.

Effect of LL-37 administration on bacterial burden in septic mice. During the course of CLP-induced sepsis, bacteria can be detected in blood as well as peritoneal cavity (23). Thus, we evaluated the effect of LL-37 administration on bacterial burden in CLP mice. A small number of bacteria were detected in blood and peritoneal fluids in the Sham group, and significantly increased in the CLP group $(\mathrm{P}<0.05)$ (Fig. 4). Notably, LL-37 inhibited the increase of the number of bacteria both in blood and peritoneal fluids $(\mathrm{P}<0.05)$. 

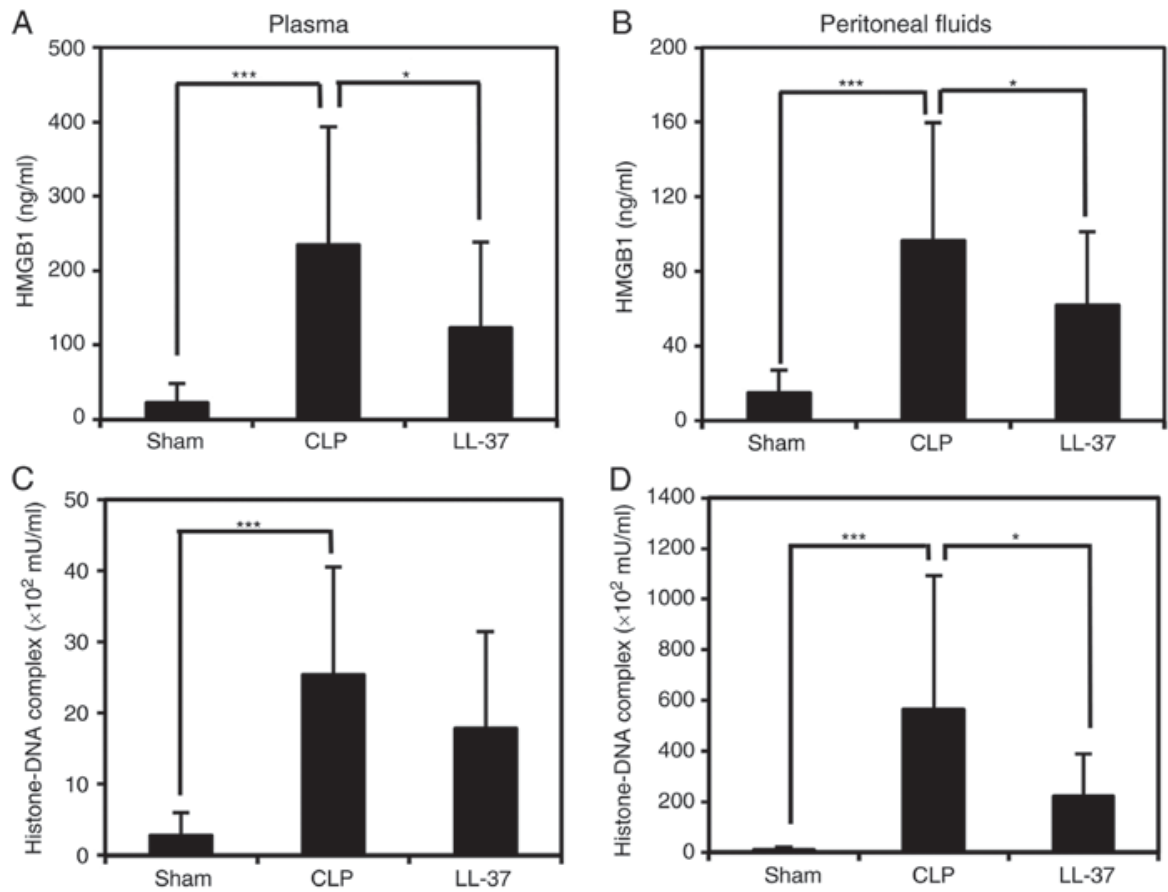

Figure 2. Effect of LL-37 administration on the levels of HMGB1 and histone-DNA complex in plasma and peritoneal fluids. Plasma and peritoneal fluids were collected from mice of Sham, CLP and LL-37 groups at $20 \mathrm{~h}$ after the surgery, and assayed for HMGB1 and histone-DNA complex. (A and B) HMGB1 levels in plasma and peritoneal fluids, respectively; (C and D) histone-DNA complex levels in plasma and peritoneal fluids, respectively. Concentrations of HMGB1 and histone-DNA complex are expressed as $\mathrm{ng} / \mathrm{ml}$ and $\mathrm{mU} / \mathrm{ml}$, respectively, in plasma and peritoneal fluids collected ( $\sim 4 \mathrm{ml})$. Data are the mean $\pm \mathrm{SD}$ of 5-19 separate experiments, and values are compared among Sham, CLP and LL-37 groups. ${ }^{*} \mathrm{P}<0.05,{ }^{* * *} \mathrm{P}<0.001$. TREM, triggering receptor expressed on myeloid; CLP, cecal ligation and puncture; HMGB1, high mobility group box 1.
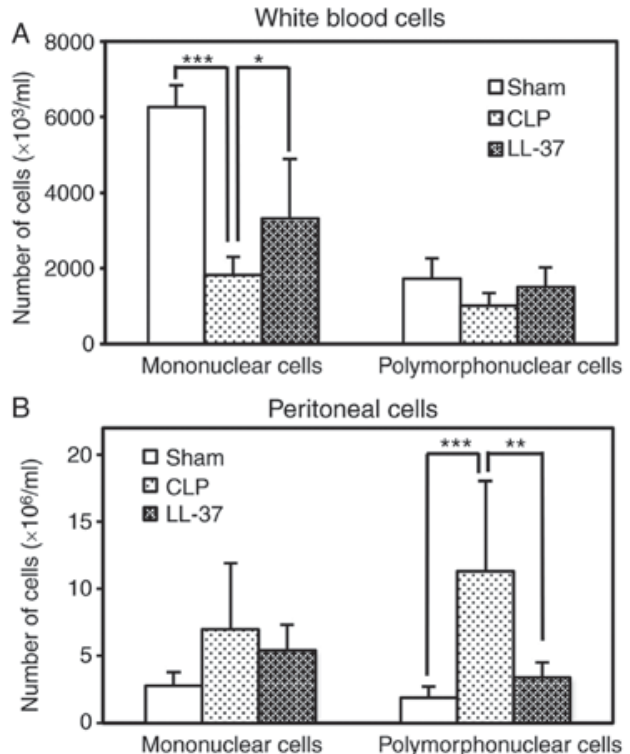

Figure 3. Effect of LL-37 administration on the numbers of white blood cells and peritoneal cells. Blood and peritoneal fluids were collected from mice of Sham, CLP and LL-37 groups at $20 \mathrm{~h}$ after the surgery, and the numbers of cells (mononuclear cells and polymorphonuclear cells) were counted. (A and B) Numbers of white blood cells and peritoneal cells, respectively. The number of mononuclear cells and polymorphonuclear cells are expressed as cells $/ \mathrm{ml}$ of blood and peritoneal fluids collected $(4 \mathrm{ml})$. Data are the mean \pm SD of 4-7 separate experiments, and values are compared among Sham, CLP and LL-37 groups. ${ }^{*} \mathrm{P}<0.05,{ }^{* *} \mathrm{P}<0.01,{ }^{* * * *} \mathrm{P}<0.001$. CLP, cecal ligation and puncture.

Effect of LL-37 administration on the levels of NETs in septic mice. During sepsis, NETs are released from activated neutrophils, and their components, such as DNA, histone and granule proteins (MPO, neutrophil elastase and proteinase 3), contribute to the pathogenesis of sepsis (14-16,27). Thus, we measured the levels of MPO-DNA complex as NETs in CLP mice, and evaluated the effect of LL-37 administration on the level of MPO-DNA complex (Fig. 5). Interestingly, MPO-DNA complex was constitutively detected in plasma of Sham group mice as well as untreated control mice (data not shown), and significantly decreased in the CLP group $(\mathrm{P}<0.001)$ (Fig. 5B). Importantly, LL-37 significantly increased the level of MPO-DNA complex in plasma $(\mathrm{P}<0.05)$. In contrast, a small amount of MPO-DNA complex was detected in peritoneal fluids in the Sham group, and the levels was significantly increased in the CLP group $(\mathrm{P}<0.001)$ (Fig. 5A). Notably, LL-37 further increased the level of MPO-DNA complex in peritoneal fluids $(\mathrm{P}<0.01)$.

Bactericidal activity of NETs. Previous reports indicated that NETs are released from neutrophils by various stimuli (such as PMA and LL-37) and exhibits bactericidal activity in vitro $(14-16,24,28,29)$. Thus, we confirmed whether NETs are released from neutrophils by PMA- or LL-37-stimulation using mouse bone marrow-derived neutrophils. The content of MPO-DNA complex (as NETs) was markedly increased by stimulation of neutrophils with $100 \mathrm{nM}$ PMA or $5 \mu \mathrm{M}$ LL-37 $(\mathrm{P}<0.05)$ (Fig. 6A).

Furthermore, we determined the bactericidal activity of NETs using the supernatants of PMA-stimulated neutrophils. Consistent with the previous reports $(38,39)$, the supernatants of PMA-stimulated neutrophils substantially exhibited the bactericidal activity compared with the supernatant of 

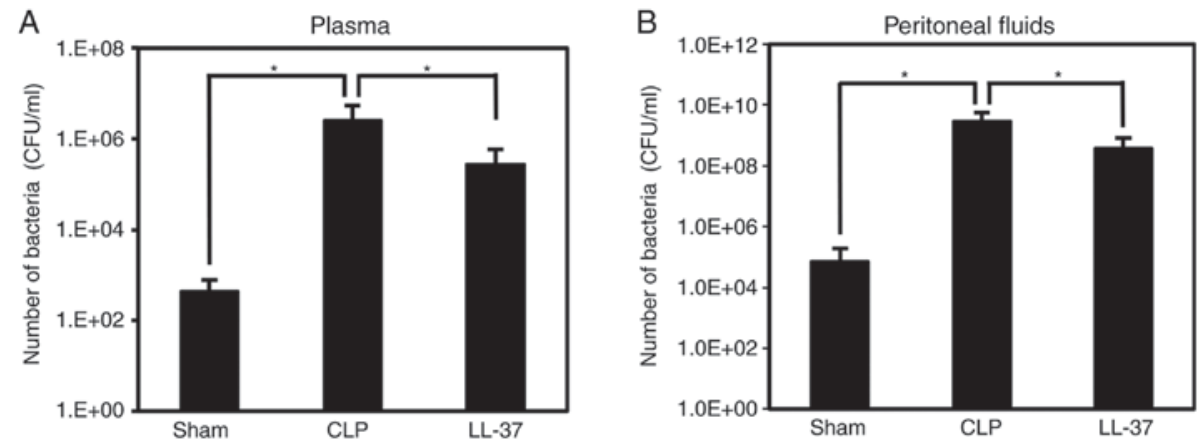

Figure 4. Effect of LL-37 administration on the bacterial burdens in blood and peritoneal fluids. Blood and peritoneal fluids were collected from mice of Sham, CLP and LL-37 groups at $20 \mathrm{~h}$ after the surgery, and serially diluted in PBS. The diluted samples were plated on Trypto-Soya agar plates, and the plates were incubated for $20 \mathrm{~h}$ at $37^{\circ} \mathrm{C}$. CFU were counted and corrected for the dilution factor. (A and B) CFU in blood and peritoneal fluids, respectively. The number of bacteria was expressed as CFU/ml of blood and peritoneal fluids collected $(\sim 4 \mathrm{ml})$. Data are the mean $\pm \mathrm{SD}$ of $6-8$ separate experiments, and values are compared among Sham, CLP and LL-37 groups. "P<0.05. CLP, cecal ligation and puncture; PBS, phosphate-buffered saline; CFU, colony forming units.
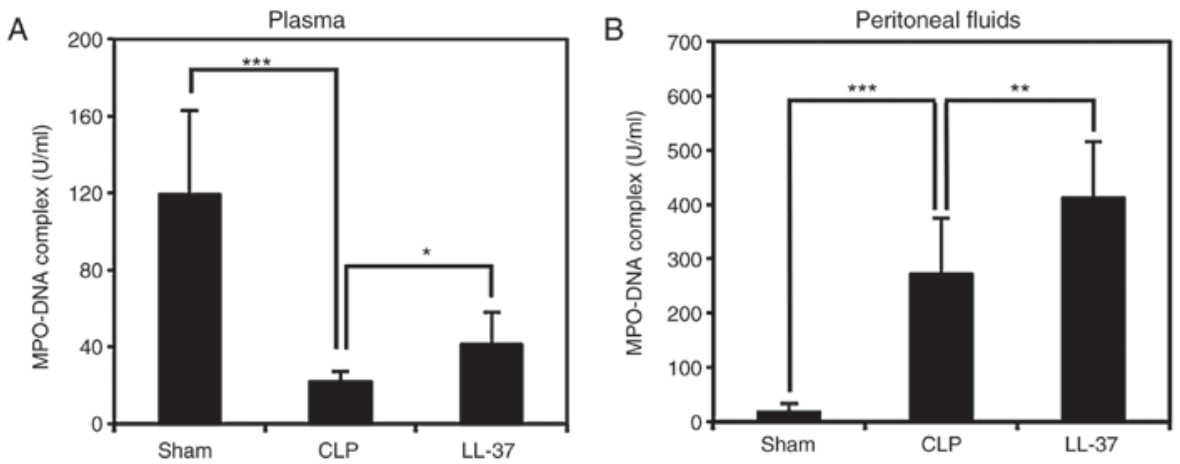

Figure 5. Effect of LL-37 administration on the levels of MPO-DNA complex in plasma and peritoneal fluids. Plasma and peritoneal fluids were collected from mice of Sham, CLP and LL-37 groups at $20 \mathrm{~h}$ after the surgery, and assayed for MPO-DNA complex. (A and B) Levels of MPO-DNA complex in plasma and peritoneal fluids, respectively. Concentrations of MPO-DNA complex are expressed as U/ml of plasma and peritoneal fluids collected ( 4 ml). Data are the mean \pm SD of 7-12 separate experiments, and values are compared among Sham, CLP and LL-37 groups. ${ }^{*} \mathrm{P}<0.05,{ }^{* *} \mathrm{P}<0.05,{ }^{* * * *} \mathrm{P}<0.001 . \mathrm{MPO}$, myeloperoxidase; CLP, cecal ligation and puncture.

A

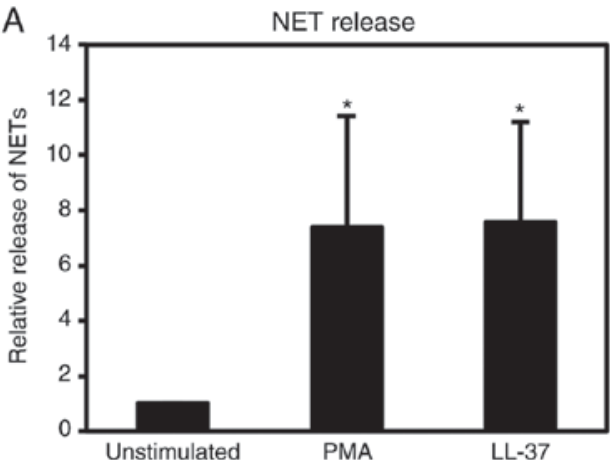

B

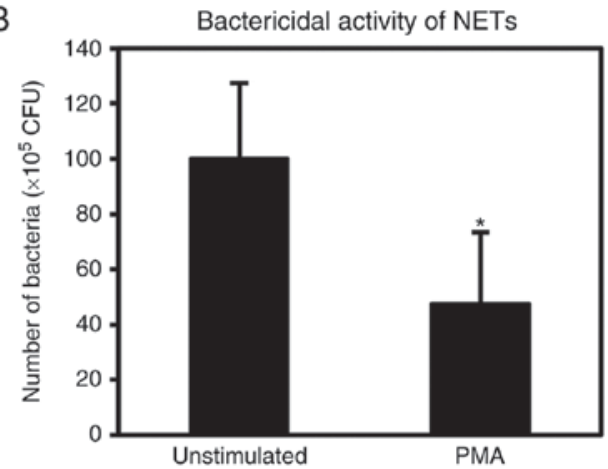

Figure 6. Induction of NET release and bactericidal activity of NETs. Mouse bone marrow-derived neutrophils $\left(10^{6}\right.$ cells) were incubated without (unstimulated) or with $100 \mathrm{nM}$ PMA or $5 \mu \mathrm{M} \mathrm{LL}-37$ for $4 \mathrm{~h}$ at $37^{\circ} \mathrm{C}$. Then, the supernatants were recovered, and assayed for MPO-DNA complex (A). Relative NET release from PMA- or LL-37-stimulated neutrophils is expressed as a ratio to that from unstimulated neutrophils. Bactericidal activity of NETs was assayed by incubating the supernatants from unstimulated or PMA-stimulated neutrophils with E. coli (10 ${ }^{7}$ cells) in LB broth at room temperature for 30 min (B). Mixtures were appropriately diluted, and plated on Trypto-Soya agar plates. The plates were incubated for $20 \mathrm{~h}$ at $37^{\circ} \mathrm{C}$, and CFU were counted and corrected for the dilution factor. Data are the mean \pm SD of 4 separate experiments, and values are compared between unstimulated and PMA- or LL-37-stimulated neutrophils. "P<0.05. NETs, neutrophil extracellular traps; PMA, phorbol 12-myristate 13-acetate; CFU, colony forming units.

unstimulated neutrophils $(\mathrm{P}<0.05)$ (Fig. 6B). In a separate experiment, we confirmed that PMA used as a stimulating agent, does not possess the bactericidal activity (data not shown).

\section{Discussion}

LL-37 is the only known member of the cathelicidin family of antimicrobial peptides in humans, cleaved from 
a precursor hCAP18 (human cationic antimicrobial polypeptide of $18-\mathrm{kDa})(7,8)$. In addition to its broad spectrum of antimicrobial activities, LL-37 can modulate various inflammatory reactions $(7,8)$. We previously revealed that LL-37 improves the survival of polybacterial CLP septic mice (23). In the present study, we further elucidated the mechanism for the protective action of LL-37 using the same CLP septic model. The results indicated that the intravenous administration of LL-37 suppressed the increase of DAMPs (such as histone-DNA complex and HMGB1) as well as TNF- $\alpha$, IL-1 $\beta$ and soluble TREM-1 in plasma and peritoneal fluids (Figs. 1 and 2). Interestingly, LL-37 significantly suppressed the decrease of mononuclear cell number in blood, and the increase of polymorphonuclear cell (neutrophil) number in the peritoneal cavity during sepsis (Fig. 3). Moreover, LL-37 reduced the bacterial burdens in blood and peritoneal fluids (Fig. 4). Notably, LL-37 increased the level of NETs (MPO-DNA complex) in plasma and peritoneal fluids (Fig. 5). In addition, we confirmed that LL-37 induces the release of NETs from neutrophils, and NETs possess the bactericidal activity (Fig. 6), consistent with the previous findings $(38,39)$. Together, these observations suggest that LL-37 improves the survival of CLP septic mice by possibly suppressing the inflammatory responses as evidenced by the inhibition of the increase of cytokines, TREM-1 and DAMPs (host cell death), the change of inflammatory cell number and the bacterial growth via the release of NETs with bactericidal activity.

Inflammatory cytokines, such as TNF- $\alpha$ and IL-1 $\beta$, function as crucial mediators for the inflammatory responses, and their serum levels correlate with the severity of sepsis; thus, inflammatory cytokines are recognized as important markers for sepsis (4). Importantly, LL-37 significantly inhibited the increase of TNF- $\alpha$ and IL-1 $\beta$ in plasma and peritoneal fluids of CLP septic mice (Fig. 1).

TREM-1 is mainly expressed on neutrophils and monocytes/macrophages and recognized as a novel receptor, which participates in the amplification of inflammatory responses, based on the findings that the silencing of TREM-1 expression and blocking of TREM-1 responses suppresses the cytokine production (IL-1 $\beta$, TNF- $\alpha$ and IL-6) and prolongs the survival of mice or rats with bacterial sepsis (40-43). Membrane-anchored TREM-1 is shed by metalloproteinases and released as soluble forms in blood (44), and soluble TREM-1 can be used as a potential marker to identify clinically ill patients with infection (30-32). The present study indicated that the levels of soluble TREM-1 are increased in plasma and peritoneal fluids of CLP septic mice, and the administration of LL-37 suppressed the increase of soluble TREM-1 levels in plasma and peritoneal fluids (Fig. 1).

DAMPs are host-cell derived biomolecules that function as potent activators of innate immune system initiating SIRS, multiple organ failure and death (45). DAMPs consist of nuclear or cytosolic molecules, such as DNA, histone, HMGB1 and ATP, and released outside the cells following tissue injury or cell death $(18-22,27,46)$. The present results indicated that the levels of histone-DNA complex and HMGB1 are increased in plasma and peritoneal fluids of CLP septic mice, and the administration of LL-37 suppressed the levels these molecules in plasma and peritoneal fluids (Fig. 2).
When exposed to bacteria or sterile stimuli, neutrophils release NETs (11). NETs are functioning as an antimicrobial mechanism by trapping microorganisms and exerting bactericidal activity via the action of NET-associated components (such as antimicrobial peptides and histone) (14-16,24,28,29). We confirmed that NETs were released from neutrophils by PMA or LL-37 stimulation, and the NET- containing supernatants exhibit the potent bactericidal activity (Fig. 6). In contrast, the major components of NETs (histone, DNA and granule proteins) are recognized as DAMPs; thus, NETs are expected to trigger inflammatory signals (18-22). In this context, we recently demonstrated that NETs themselves do not induce the IL-1 $\beta$ production by macrophages but augment the IL-1 $\beta$ production in combination with LPS, and NET-associated DNA and serine proteases are involved in NET/LPS-induced IL-1 $\beta$ production as essential components (27). Interestingly, however, the LL-37 administration increased the levels of NETs, but decreased the levels of IL-1 $\beta$ in plasma and peritoneal fluids (Figs. 1 and 5). LL-37 can bind with LPS and neutralize the activity of LPS $(11,25)$. Thus, it could be speculated in our CLP model that LL-37 neutralized the action of LPS and suppresses the NET-mediated IL-1 $\beta$ production. Conversely, based on its NET-releasing activity (24), LL-37 is expected to induce the release of NETs with bactericidal activity in vivo, thereby suppressing bacterial burdens and improving the inflammatory responses of sepsis, as evidence by the inhibition of the increase of cytokines, TREM-1 and DAMPs (host cell death), and the change of inflammatory cell number. Interestingly, the concentration of plasma LL-37 is estimated to be $>1 \mu \mathrm{g} / \mathrm{ml}$ (LL-37, $2 \mu \mathrm{g}$ per mouse; body weight, $\sim 23 \mathrm{~g}$ ), and we confirmed that LL-37 can substantially induce the release of NETs from bone marrow-derived neutrophils in vitro at $0.5 \mu \mathrm{M}(2 \mu \mathrm{g} / \mathrm{ml})$ in preliminary experiments (data not shown); these observations support the possibility that LL-37 induces the release of NETs in vivo. Moreover, recent studies have indicated that NETs serve as a scaffold for thrombus formation in sepsis $(47,48)$, and intravascular thrombosis (immunothrombosis), which is related to innate immune mechanism, plays a key role in the suppression of pathogen spreading and the restriction of pathogen survival during infection (48). Thus, it is possible that LL-37-induced NETs not only exhibits the bactericidal activity but also suppress the pathogen spreading and persistence via immunothrombosis, thereby relieving the inflammatory reactions and improving the survival of CLP septic mice. However, LL-37 itself is also expected to exert the bactericidal activity in vivo, because LL-37 exhibits an antimicrobial activity in vitro [minimum inhibitory concentration (MIC), 0.1 10 $\mu \mathrm{g} / \mathrm{ml}]$ (7).

Notably, NETs were released abundantly in the peritoneal cavity compared with blood by LL-37 administration (Fig. 5). Correspondingly, LL-37 administration more potently suppressed the increase of bacterial burden, cytokines (TNF- $\alpha$ and IL-1 $\beta$ ), soluble TREM-1, DAMPs (especially histone-DNA complex) and inflammatory cell recruitment in the peritoneal cavity compared with blood (Figs. 1-4). Thus, NETs likely exhibit the protective action on the inflammatory reactions at the local milieu (the site of infection and inflammation) in the CLP model, when released extracellularly.

Recently, we have examined the effect of LL-37 on pyroptosis, a caspase-1-dependent cell death, which mainly occurs in 
macrophages, accompanied with the release of pro-inflammatory cytokines (such as IL-1 $\beta$ ), and revealed that LL-37 improves the survival of CLP septic mice by suppressing the pyroptosis of macrophages, inflammatory cytokine production by activated macrophages and bacterial growth (27). In the present study, to elucidate the protective actions of LL-37 on sepsis, we focused on the effect of LL-37 on NET release, and indicated that LL-37 improves the survival of CLP septic mice by suppressing the inflammatory responses and bacterial growth possibly via the release of NETs with bactericidal activity. These observations suggest that LL-37 protects CLP septic mice through at least two mechanisms, i.e., the inhibition of pyroptosis and the release of NETs (induction of NETosis). Thus, LL-37 can be a promising therapeutic candidate for sepsis because of its various functions, including the modulation of cells death (pyroptosis and NETosis), inflammatory cytokine production and bactericidal activity.

\section{Acknowledgements}

We are grateful to Drs Taisuke Murakami and Kaori Suzuki (Department of Host Defense and Biochemical Research, Juntendo University, Graduate School of Medicine) for the helpful suggestion and discussion. This work was supported in part by a Grant-in-Aid for Young Scientists B (grant no. 24790424) and a Grant-in-Aid (grant nos. 26460538 and 17K08840) for Scientific Research from Japan Society for the Promotion of Science, a Grant-in-Aid (grant no. S0991013) and a Grants-in-Aid (grant no. S1201013) from the Ministry of Education, Culture, Sports, Science and Technology, Japan (MEXT) for the Foundation of Strategic Research Projects in Private Universities. The authors thank Center for Biomedical Research Resources, Juntendo University Graduate School of Medicine.

\section{References}

1. Bone RC: The pathogenesis of sepsis. Ann Intern Med 115: 457-469, 1991.

2. Angus DC: The search for effective therapy for sepsis: Back to the drawing board? JAMA 306: 2614-2615, 2011.

3. O'Brien JM Jr, Ali NA, Aberegg SK and Abraham E: Sepsis. Am J Med 120: 1012-1022, 2007.

4. Pinsky MR: Dysregulation of the immune response in severe sepsis. Am J Med Sci 328: 220-229, 2004.

5. Pinheiro da Silva F and Nizet V: Cell death during sepsis: Integration of disintegration in the inflammatory response to overwhelming infection. Apoptosis 14: 509-521, 2009.

6. Hotchkiss RS and Nicholson DW: Apoptosis and caspases regulate death and inflammation in sepsis. Nat Rev Immunol 6: 813-822, 2006.

7. Dürr UH, Sudheendra US and Ramamoorthy A: LL-37, the only human member of the cathelicidin family of antimicrobial peptides. Biochem Biophys Acta 1758: 1408-1425, 2006.

8. Hancock RE and Diamond G: The role of cationic antimicrobial peptides in innate host defences. Trtends Microbiol 8: 402-410, 2000.

9. Suzuki K, Murakami T, Kuwahara-Arai K, Tamura H, Hiramatsu K and Nagaoka I: Human anti-microbial cathelicidin peptide LL-37 suppresses the LPS-induced apoptosis of endothelial cells. Int Immunol 23: 185-193, 2011.

10. Nagaoka I, Tamura $\mathrm{H}$ and Hirata $\mathrm{M}$ : An antimicrobial cathelicidin peptide, human CAP18/LL-37, suppresses neutrophil apoptosis via the activation of formyl-peptide receptor-like 1 and P2X7. J Immunol 176: 3044-3052, 2006.

11. Nagaoka I, Hirota S, Niyonsaba F, Hirata M, Adachi Y, Tamura H, Tanaka S and Heumann D: Augmentation of the lipopolysaccharide-neutralizing activities of human cathelicidin CAP18/LL-37-derived antimicrobial peptides by replacement with hydrophobic and cationic amino acid residues. Clin Diagn Lab Immunol 9: 972-982, 2002.
12. Mantovani A, Cassatella MA, Costantini C and Jaillon S: Neutrophils in the activation and regulation of innate and adaptive immunity. Nat rev Immunol 11: 519-531, 2011.

13. Nathan N: Neutrophils and immunity: Challenges and opportunities. Nat Rev Immunol 6: 173-182, 2006.

14. Camicia G, Pozner R and de Larrañaga G: Neutrophil extracellular traps in sepsis. Shock 42: 286-294, 2014.

15. Remijsen Q, Kuijpers TW, Wirawan E, Lippens S, Vandenabeele P and Vanden Berghe T: Dying for a cause: NETosis, mechanisms behind an antimicrobial cell death modality. Cell Death Differ 18: 581-588, 2011.

16. McDonald B, Urrutia R, Yipp BG, Jenne CN and Kubes P: Intravascular neutrophil extracellular traps capture bacteria from the bloodstream during sepsis. Cell Host Microbe 12: 324-333, 2012.

17. Kawasaki $\mathrm{H}$ and Iwamuro S: Potential roles of histones in host defense as antimicrobial agents. Infect Disord Drug Targets 8: 195-205, 2008.

18. Urban CF, Ermert D, Schmid M, Abu-Abed U, Goosmann C, Nacken W, Brinkmann V, Jungblut PR and Zychlinsky A: Neutrophil extracellular traps contain calprotectin, a cytosolic protein complex involved in host defense against Candida albicans. PLos Pathog 5: e1000639, 2009.

19. Saffarzadeh M, Juenemann C, Queisser MA, Lochnit G, Barreto G, Galuska SP, Lohmeyer J and Preissner KT: Neutrophil extracellular traps directly induce epithelial and endothelial cell death: A predominant role of histones. PLos One 7: e32366, 2012.

20. Alfaidi M, Wilson H, Daigneault M, Burnett A, Ridger V, Chamberlain $\mathrm{J}$ and Francis S: Neutrophil elastase promotes interleukin-1 $\beta$ secretion from human coronary endothelium. J Biol Chem 290: 24067-24078, 2015.

21. Xu J, Zhang X, Monestier M, Esmon NL and Esmon CT: Extracellular histones are mediators of death through TLR2 and TLR4 in mouse fatal liver injury. J Immunol 187: 2626-2631, 2011.

22. Xu J,Zhang X, Pelayo R, Monestier M, Ammollo CT, Semeraro F, Taylor FB, Esmon NL, Lupu F and Esmon CT: Extracellular histones are major mediators of death in sepsis. Nat Med 15: 1318-1321, 2009.

23. Hu Z, Murakami T, Suzuki K, Tamura H, Reich J, Kuwahara-Arai K, Iba T and Nagaoka I: Antimicrobial cathelicidin peptide LL-37 inhibits the pyroptosis of macrophages and improves the survival of polybacterial septic mice. Int Immunol 28: 245-253, 2016.

24. Neumann A, Berends ET, Nerlich A, Molhoek EM, Gallo RL, Meerloo T, Nizet V, Naim HY and von Köckritz-Blickwede M: The antimicrobial peptide LL-37 facilitates the formation of neutrophil extracellular traps. Biochem J 464: 3-11, 2014.

25. Nagaoka I, Hirota S, Niyonsaba F, Hirata M, Adachi Y, Tamura H and Heumann D: Cathelicidin family of antibacterial peptides CAP18 and CAP11 inhibit the expression of TNF- $\alpha$ by blocking the binding of LPS to CD14(+) cells. J Immunol 167: 3329-3338, 2001.

26. Hubbard WJ, Choudhry M, Schwacha MG, Kerby JD, Rue LW III, Bland KI and Chaudry IH: Cecal ligation and puncture. Shock 24 (Suppl 1): S52-S57, 2005.

27. Hu Z, Murakami T, Tamura H, Reich J, Kuwahara-Arai K, Iba T, Tabe Y and Nagaoka I: Neutrophil extracellular traps induce IL-1 $\beta$ production by macrophages in combination with lipopolysaccharide. Int J Mol Med 39: 549-558, 2017.

28. Sil P, Yoo DG, Floyd M, Gingerich A and Rada B: High throughput measurement of extracellular DNA release and quantitative NET formation in human neutrophils in vitro. J Vis Exp: Jun 18, 2016 (Epub ahead of print). doi: 10.3791/52779.

29. Yoo DG, Floyd M, Winn M, Moskowitz SM and Rada B: NET formation induced by Pseudomonas aeruginosa cystic fibrosis isolates measured as release of myeloperoxidase-DNA and neutrophil elastase-DNA complexes. Immunol Lett 160: 186-194, 2014.

30. Gibot S, Kolopp-Sarda MN, Béné MC, Cravoisy A, Levy B, Faure GC and Bollaert PE: Plasma level of a triggering receptor expressed on myeloid cells-1: Its diagnostic accuracy in patients with suspected sepsis. Ann Intern Med 141: 9-15, 2004.

31. Quenot JP, Luyt CE, Roche N, Chalumeau M, Charles PE, Claessens YE, Lasocki S, Bedos JP, Péan Y, Philippart F, et al: Role of biomarkers in the management of antibiotic therapy: An expert panel review II: Clinical use of biomarkers for initiation or discontinuation of antibiotic therapy. Ann Intensive Care 3: $21,2013$. 
32. Charles PE, Noel R, Massin F, Guy J, Bollaert PE, Quenot JP and Gibot S: Significance of soluble triggering receptor expressed on myeloid cells-1 elevation in patients admitted to the intensive care unit with sepsis. BMC Infect Dis 16: 559, 2016.

33. Huang H, Evankovich J, Yan W, Nace G, Zhang L, Ross M, Liao X, Billiar T, Xu J, Esmon CT and Tsung A: Endogenous histones function as alarmins in sterile inflammatory liver injury through Toll-like receptor 9 in mice. Hepatology 54: 999-1008, 2011.

34. Huebener P, Pradere JP, Hernandez C, Gwak GY, Caviglia JM, Mu X, Loike JD, Jenkins RE, Antoine DJ and Schwabe RF: The HMGB1/RAGE axis triggers neutrophil-mediated injury amplification following necrosis. J Clin Invest 125: 539-550, 2015.

35. Chen R, Fu S, Fan XG, Lotze MT, Zeh HJ III, Tang D and Kang R: Nuclear DAMP complex-mediated RAGE-dependent macrophage cell death. Biochem Biophys Res Commun 458: 650-655, 2015

36. Shi $\mathrm{C}$ and Pamer EG: Monocyte recruitment during infection and inflammation. Nat Rev Immunol 11: 762-774, 2011.

37. Kolaczkowska E and Kubes P: Neutrophil recruitment and function in health and inflammation. Nat Rev Immunol 13: 159-175, 2013.

38. Brinkmann V, Reichard U, Goosmann C, Fauler B, Uhlemann Y, Weiss DS, Weinrauch Y and Zychlinsky A: Neutrophil extracellular traps kill bacteria. Science 303: 1532-1535, 2004.

39. Gorgojo J, Scharrig E, Gómez RM,Harvill ET and Rodríguez ME: Bordetella parapertussis circumvents neutrophil extracellular bactericidal mechanisms. PLoS One 12: e0169936, 2017.

40. Bouchon A, Facchetti F, Weigand MA and Colonna M: TREM-1 amplifies inflammation and is a crucial mediator of septic shock. Nature 410: 1103-1107, 2001.
41. Gibot S, Alauzet C, Massin F, Sennoune N, Faure GC, Béné MC, Lozniewski A, Bollaert PE and Lévy B: Modulation of the triggering receptor expressed on myeloid cells-1 pathway during pneumonia in rats. J Infect Dis 194: 975-983, 2006.

42. Gibot S, Buonsanti C, Massin F, Romano M, Kolopp-Sarda MN, Benigni F, Faure GC, Béné MC, Panina-Bordignon P, Passini N and Lévy B: Modulation of the triggering receptor expressed on the myeloid cell type 1 pathway in murine septic shock. Infect Immun 74: 2823-2830, 2006.

43. Gibot S, Massin F, Marcou M, Taylor V, Stidwill R, Wilson P, Singer $\mathrm{M}$ and Bellingan G: TREM-1 promotes survival during septic shock in mice. Eur J Immunol 37: 456-466, 2007.

44. Gómez-Piña V, Soares-Schanoski A, Rodríguez-Rojas A, Del Fresno C, García F, Vallejo-Cremades MT, Fernández-Ruiz I, Arnalich F, Fuentes-Prior P and López-Collazo E: Metalloproteinases shed TREM-1 ectodomain from lipopolysaccharide-stimulated human monocytes. J Immunol 179: 4065-4073, 2007.

45. Matzinger P: The danger model: A renewed sense of self. Science 296: 301-305, 2002.

46. Bergsbaken T, Fink SL and Cookson BT: Pyroptosis: Host cell death and inflammation. Nat Rev Microbiol 7: 99-109, 2009.

47. Iba T, Hashiguchi N, Nagaoka I, Tabe Y and Murai M: Neutrophil cell death in response to infection and its relation to coagulation. J Intensive Care 1: 13, 2013.

48. Engelmann B and Massberg S: Thrombosis as an intravascular effector of innate immunity. Nat Rev Immunol 13: 34-45, 2013. 